\section{How Are Rheumatologists Managing Anticyclic Citrullinated Peptide Antibodies-positive Patients Who Do Not Have Arthritis?}

To the Editor:

Early referral and initiation of disease-modifying antirheumatic drugs (DMARD) is associated with better outcomes for patients with rheumatoid arthritis (RA) ${ }^{1,2}$. In the United Kingdom, general practitioners (GP) are advised to refer patients with suspected RA urgently ${ }^{3}$ and rheumatology departments are rewarded for timely management of these patients ${ }^{4}$.

Although a positive step, a corollary of this is that rheumatologists are now seeing patients earlier in the natural history of RA [e.g., patients with autoantibodies, especially anticyclic citrullinated peptide antibodies (anti-CCP) and symptoms but no clinical synovitis, who are at risk of developing RA]. This presents a clinical problem but also a significant opportunity. There is no evidence for the management of these (often symptomatic) at-risk individuals, but it is possible that the right intervention in this phase may prevent clinical arthritis ${ }^{5,6}$. This hypothesis is being explored in clinical trials (e.g., rituximab delayed, but did not prevent, arthritis onset in at-risk individuals) ${ }^{7}$. We were interested in how such at-risk individuals are managed by UK rheumatologists in the absence of guidelines. We were specifically interested in the use of imaging and hypothesized that rheumatologists use imaging to guide their management.

We conducted a survey by circulating anonymous questionnaires at a national meeting for clinical rheumatologists that focused on new developments in rheumatology (Revolutions in Rheumatology 2018, London, UK) and at regional general rheumatology meetings in Yorkshire, UK (survey questions available from the authors on request). Questionnaires were returned from 47 consultant rheumatologists working in 39 different UK hospitals (excluding Leeds).

The vast majority of respondents $(44 / 47 ; 94 \%)$ reported that they are referred anti-CCP-positive $(\mathrm{CCP}+)$ patients who have musculoskeletal symptoms but no clinical synovitis in their routine clinical practice. Of these, $32 / 44(73 \%)$ were referred $>5$ patients per year. In CCP+ patients with inflammatory symptoms but no clinical synovitis, 36/44 (82\%) said they would request an ultrasound (US) scan to help guide management. Five percent (2/44) would request a magnetic resonance imaging scan. All respondents said they would follow up these patients regularly and 5/44 $(11 \%)$ would consider a clinical trial. In CCP+ patients with noninflammatory symptoms and no clinical synovitis, 12/44 (27\%) would discharge them back to primary care, whereas $18 / 44$ (41\%) would request an US scan and $13 / 44(30 \%)$ would observe in clinic.

Rheumatologists were then asked, "Do you use imaging to help guide your management of anti-CCP-positive patients without clinical synovitis?" A majority, 40/44 (91\%), reported they used imaging, with most (37/40, 93\%) using US. For patients where power Doppler (PD) signal is present on US in at least 1 joint, the majority of respondents $(27 / 37,73 \%)$ would start treatment, usually a DMARD [corticosteroid alone, 6/27 (22\%); methotrexate (MTX), 8/27 (30\%); hydroxychloroquine (HCQ), 11/27 (41\%); either MTX or HCQ, $2 / 27(7 \%)$ ]. There were 6/37 (15\%) respondents who would simply treat according to their standard RA pathway. The remainder would observe without treatment or consider a clinical trial.

In patients with US tenosynovitis but no US synovitis, 26/37 (70\%) would treat, the majority with corticosteroids alone (13/26, 50\%) compared to DMARD [MTX, 3/26 (12\%); HCQ, 10/26 (38\%)]. In patients with no US synovitis or US tenosynovitis, 23/37 (62\%) would observe without therapy while 12/37 (32\%) would discharge the patient (Table 1).

Finally, 35/45 (78\%) respondents said formal guidance on how to manage anti-CCP+ individuals without clinical synovitis would be useful.

These data suggest UK rheumatologists see anti-CCP+ patients without clinical arthritis in routine practice, and in the absence of guidelines, use clinical intuition and US findings to guide management. Interestingly, US was used by $84 \%$ of respondents. Further, the pattern of US inflammation appears to influence the choice of treatment; patients with US synovitis (i.e., PD in the joints) receive the most intensive therapy, with $71 \%$ either treated
Table 1. The management of anti-CCP-positive individuals without clinical synovitis according to ultrasound findings, as reported in a survey of consultant rheumatologists in the United Kingdom.

Management

Ultrasound Findings, $\mathrm{n}=37$

Power Doppler Tenosynovitis No Synovitis or Signal in $\geq 1 \quad$ Only, $\mathrm{n}(\%) \quad$ Tenosynovitis,

Joint, $\mathrm{n}(\%) \quad \mathrm{n}(\%)$

\begin{tabular}{|c|c|c|c|}
\hline Treat as RA & $6(16)$ & $0(0)$ & $0(0)$ \\
\hline MTX monotherapy & $8(21)$ & $3(8)$ & $0(0)$ \\
\hline MTX or HCQ monotherapy & $2(5)$ & $0(0)$ & $0(0)$ \\
\hline HCQ monotherapy & $11(29)$ & $10(27)$ & $2(5)$ \\
\hline Corticosteroids only & $6(16)$ & $13(35)$ & $0(0)$ \\
\hline $\begin{array}{l}\text { Observe in clinic } \\
\text { (no treatment) }\end{array}$ & $1(3)$ & $8(21)$ & $23(62)$ \\
\hline $\begin{array}{l}\text { Discharge from clinic } \\
\text { (no treatment) }\end{array}$ & $0(0)$ & $0(0)$ & $12(32)$ \\
\hline Consider for clinical trial & $3(8)$ & $3(8)$ & $0(0)$ \\
\hline
\end{tabular}

Anti-CCP: anticyclic citrullinated peptide antibodies; RA: rheumatoid arthritis; MTX: methotrexate; HCQ: hydroxychloroquine.

as a standard patient with RA or given a DMARD. In contrast, $94 \%$ of patients with no US inflammation are either observed in clinic without therapy or discharged. There is certainly some logic to this approach; US is a readily available, noninvasive test, and the presence of PD has been shown to predict clinical arthritis development in anti-CCP+ patients without clinical synovitis ${ }^{8}$.

However, there are many unanswered questions: for example, should US or other serological biomarkers be used to stratify for treatment intensity? Are synthetic DMARD an appropriate choice in the "preclinical arthritis" phase of RA? If so, what is the optimum treatment regimen? What are rheumatologists' views on RA prevention, both in the UK and worldwide? Clearly these and other questions must be addressed through research and it is encouraging that some of the surveyed rheumatologists considered these patients for clinical trials.

Our survey suggests that rheumatologists are seeing patients in the preclinical arthritis phase of RA and often using DMARD, guided by US findings. Whether this pragmatic approach is an appropriate one should now be tested in optimally designed clinical trials, with RA prevention the ultimate ambition.

KULVEER MANKIA @ , DM, MRCP, Leeds Institute of Rheumatic and Musculoskeletal Medicine, University of Leeds, and UK National Institute for Health Research (NIHR) Leeds Biomedical Research Centre, Leeds Teaching Hospitals National Health Service (NHS) Trust; CHRISTOPHER BRIGGS, MBBS, Leeds Teaching Hospitals NHS Trust; PAUL EMERY (1), MD, FRCP, Leeds Institute of Rheumatic and Musculoskeletal Medicine, University of Leeds, and UK NIHR Leeds Biomedical Research Centre, Leeds Teaching Hospitals NHS Trust, Leeds, UK. Address correspondence to Dr. K. Mankia, Academic Clinical Lecturer in Rheumatology, Leeds Institute of Rheumatic and Musculoskeletal Medicine, Chapel Allerton Hospital, Chapeltown Road, Leeds LS7 4SA, UK. E-mail: k.s.mankia@leeds.ac.uk

\section{REFERENCES}

1. Quinn MA, Emery P. Window of opportunity in early rheumatoid arthritis: possibility of altering the disease process with early intervention. Clin Exp Rheumatol 2003;21:S154-7.

2. Nell VP, Machold KP, Eberl G, Stamm TA, Uffmann M, Smolen JS. Benefit of very early referral and very early therapy with disease-modifying anti-rheumatic drugs in patients with early rheumatoid arthritis. Rheumatology 2004;43:906-14. 
3. Rheumatoid arthritis in adults: management. NICE guideline [NG100]. [Internet. Accessed October 10, 2019.] Available from: www.nice.org.uk/guidance/ng 100

4. Best practice tariff: early inflammatory arthritis. 2019/20 national tariff. Tariff documents. Annex F: Models used to calculate prices. [Internet. Accessed November 20, 2019.] Available from: improvement.nhs.uk

5. Mankia K, Emery P. A new window of opportunity in rheumatoid arthritis: targeting at-risk individuals. Curr Opin Rheumatol 2016;28:260-6.

6. Cope AP. Emerging therapies for pre-RA. Best Pract Res Clin Rheumatol 2017;31:99-111.

7. Gerlag DM, Safy M, Maijer KI, Tang MW, Tas SW, Starmans-Kool MJF, et al. Effects of B-cell directed therapy on the preclinical stage of rheumatoid arthritis: the PRAIRI study. Ann Rheum Dis 2019;78:179-85.

8. Nam JL, Hensor EM, Hunt L, Conaghan PG, Wakefield RJ, Emery P. Ultrasound findings predict progression to inflammatory arthritis in anti-CCP antibody-positive patients without clinical synovitis. Ann Rheum Dis 2016;75:2060-7.

First Release December 1 2019; J Rheumatol 2020;47:2;

doi:10.3899/jrheum.190211 